\title{
PROXIMITY SPACES AND TOPOLOGICAL FUNCTORS
}

\author{
W. N. HUNSAKER AND P. L. SHARMA
}

ABSTRACT. The purpose of this paper is to determine what natural functors $T: A \rightarrow X$ are $(\mathcal{E}, \pi)$-topological, where $A$ is a subcategory of the category of proximity or uniform spaces and $X$ is an $(\tilde{\xi}, \pi)$-category. We give necessary and sufficient conditions under which a point separating family of continuous functions can be nicely lifted to a proximally continuous family. Proximities having a finest compatible uniform structure are characterized.

Introduction. A problem which frequently arises in analys is is to determine whether or not a given continuous function is proximally (uniformly) continuous. More generally, let $X$ be a topological space and let $F$ be a family of continuous functions, each member $f$ of $F$ being from $X$ into a proximity space $Y_{f}$. Does there exist a "compatible" proximity structure $\delta$ on $X$ satisfying:

(i) each $f \in F$ is $p$-continuous on $(X, \delta)$, and

(ii) for any proximity space $Z$ and any continuous function $g$ from $Z$ to $X, g$ is $p$-continuous iff $f g$ is $p$-continuous for each $f \in F$.

Problems similar to this are solved in standard textbooks on topology with $X$ a set, $F$ a family of functions and $Y_{f}$ either a uniform space or a topological space; however, more general problems of this type have not yet been discussed in the literature.

In order to study these questions it is convenient to make use of the notions of $(\mathcal{E}, \mathbb{N})$-categories and topological functors as introduced by Herrlich [2].

1. Preliminaries. Let $\mathbf{X}$ be a category. A source in $\mathbf{X}$ is a pair $\left(X, f_{i}\right)$, where $X$ is an object in $\mathbf{X}$ and $f_{i}: X \rightarrow X_{i}$ is a family of $X$-morphisms indexed by a class $I .\left(X, f_{i}\right)_{I}$ is a monosource if it is a source, and $r=s$ whenever $f_{i} r=f_{i} s$ for every $i \in I$. A morphism $e$ in a category $\mathbf{X}$ is a regular epimor-

Received by the editors June 1, 1973 and, in revised form, October 12, 1973.

AMS (MOS) subject classifications (1970). Primary 54A10, 54E05, 54E15, 18B99; Secondary 18A40, 54C99.

Key words and phrases. Source, absolutely topological functor, $L O$-proximity, $E F$-proximity, uniformity, proximity class, weak proximity.

Copyright $\odot$ 1974, American Mathematical Society 
phis $m$ if and only if it is the coequalizer of some pair of morphisms in $\mathbf{X}$. A category $X$ is an $(\mathscr{E}, \mathbb{N})$-category provided that $\mathcal{E}$ is a class of epimorphisms in $X$, closed under composition with isomorphisms, $\mathbb{N}$ is a class of sources in $X$, closed under composition with isomorphisms, and the following conditions hold:

(a) For every source $\left(X, f_{i}\right)_{I}$ in $X$ there exists $e$ in $\mathscr{E}$ and $\left(Y, m_{i}\right)_{I}$ in $\mathbb{M}$ such that $f_{i}=m_{i} e$ for each $i \in I$, and

(b) whenever $f$ and $e$ are morphisms and $\left(Y, m_{i}\right)_{I}$ and $\left(Z, f_{i}\right)_{I}$ are sources in $X$ such that $e \in \mathcal{E},\left(Y, m_{i}\right) \in \mathbb{M}$ and $f_{i} e=m_{i} f$ for each $i \in I$, then there exists a (unique) morphism $g$ in $X$ such that $f=g e$ and $f_{i}=m_{i} g$ for each $i \in I$.

(1.1) Definition. Let $\mathbf{X}$ be an $(\mathcal{E}, \mathbb{M})$-category and let $T: \mathbf{A} \rightarrow \mathbf{X}$ be a functor.

(i) A source $\left(A, f_{i}\right)_{I}$ in $\mathbb{A}^{N}$ is called $T$-initial provided for any source $\left(B, g_{i}\right)$ in $\mathbf{A}$ and any $\mathbf{X}$-morphism $f: T B \rightarrow T A$ with $T f_{i} \cdot f=T g_{i}$, for each $i \in I$, there exists a unique $\mathbf{A}$-morphis $b: B \rightarrow A$ in $\mathbf{A}$ such that $T h=f$ and $f_{i} h=g_{i}$ for each $i \in I$.

(ii) A source $\left(A, f_{i}: A \rightarrow A_{i}\right)_{I} T$-lifts a source $\left(X, g_{i}: X \rightarrow T A_{i}\right)_{I}$ in $\mathbf{X}$ if and only if there exists an isomorphism $k: X \rightarrow T A$ in $X$ with $T f_{i} k=g_{i}$ for each $i \in I$.

(iii) A source $\left(A, f_{i}\right)_{I}$ in $\mathbf{A}$ is initial to a source $\left(X, T f_{i}\right)_{I}$ in $X$ if and only if $\left(A, f_{i}\right)_{I}$ is $T$-initial and $T$-lifts $\left(X, T f_{i}\right)_{I^{\bullet}}$. When the meaning is clear from the context, we say that $A$ is initial to the source $\left(X, T f_{i}\right)_{I^{\bullet}}$.

(iv) $T$ is $(\mathcal{E}, \mathcal{M})$-topological if and only if for each family $\left(A_{i}\right)_{I}$ of Aobjects and each source $\left(X, m_{i}: X \rightarrow T A_{i}\right)_{I}$ in $\mathbb{M}$ there exists a $T$-initial source $\left(A, f_{i}: A \rightarrow A_{i}\right)_{I}$ in $\mathbf{A}$ which $T$-lifts $\left(X, m_{i}\right)_{I^{*}}$

(v) $T$ is called absolutely topological if and only if it is $(\mathscr{G}, \mathbb{M}$ )-topological for any $(\mathcal{E}, \mathbb{M}$ )-structure on $\mathbf{X}$.

For the definitions of $L O$ - and $E F$-proximities, the reader is referred to [1]. The definition of a $L O$-base is analogous to that of an $E F$-base given in [5].

If $\delta$ is a $L O$-proximity on $X$ then $(X, \delta)$ is called a $L O$-space (respectively an $E F$-space). For two $L O$-spaces $(X, \theta)$ and $(Y, \delta)$, a function $f$ on $X$ to $Y$ is said to be $p$-continuous provided $(A, B) \in \theta$ implies $(f A, f B) \in \delta$.

For two binary relations $\theta_{1}$ and $\theta_{2}$ on $\mathcal{P}(X)$, we say that $\theta_{1}$ is finer than $\theta_{2}\left(\theta_{2}\right.$ is coarser than $\left.\theta_{1}\right)$ if and only if $\theta_{1} \subset \theta_{2}$. For a given LO-space $(X, \delta)$ and a subset $A \subset X$, defining $\bar{A}=\{x \in X:(\{x\}, A) \in \delta\}$ we get a Kuratowski closure operator on $X$. The induced topology is always $R_{0}$. For a 
$R_{0}$-space $(X, \mathcal{J})$ there are two distinguished $L O$-proximities. The finest $L O$-proximity which induces $\mathcal{T}$ is denoted by $\delta_{0}$ and is given by $(A, B) \in \delta_{0}$ if and only if $\bar{A} \cap \bar{B} \neq \varnothing$. A subset of the form $\{\overline{x\}}$ is called a point closure. The coarsest $L O$-proximity inducing $\mathcal{J}$ is denoted by $\delta_{c}$ and is given by: $(A, B) \in \delta_{c}$ if and only if $\bar{A} \cap \bar{B} \neq \varnothing$ or each of the sets $\bar{A}$ and $\bar{B}$ is a union of infinitely many distinct point-closures [4].

Suppose $\beta$ is a $L O$-base on a set $X$. Define $\delta(\beta)$ as follows: $(A, B) \notin$ $\delta(\beta)$ if and only if there are finite covers $\left\{A_{i}: 1 \leqq i \leqq n\right\}$ and $\left\{B_{j}: 1 \leqq j \leqq m\right\}$ of $A$ and $B$ respectively such that $\left(A_{i}, B_{j}\right) \notin \beta$ for any $i, j$. Then $\delta(\beta)$ is the coarsest $L O$-proximity finer than the base $\beta$. The $L O$-proximity (respectively $E F$-proximity) $\delta(\beta)$ is said to be generated by the base $\beta$. Note that if $\beta$ is an $E F$-base then $\delta(\beta)$ is an $E F$-proximity.

(1.3) Lemma. Let $\beta_{1}, \beta_{2}$ be LO-bases on sets $X$ and $Y$ respectively, and let $f$ be a function on $X$ to $Y$ such that $(A, B) \in \beta_{1}$ implies $(f A, f B) \epsilon$ $\beta_{2}$. Then $f:\left(X, \delta\left(\beta_{1}\right)\right) \rightarrow\left(Y, \delta\left(\beta_{2}\right)\right)$ is p-continuous.

For any collection $S=\left\{\delta_{i}: i \in I\right\}$ of $L O$-proximities on a set $X, \bigvee S$ denotes the join of the collection $S$. If each $\delta_{i}$ is $E F$ then so is $\bigvee \delta_{i}$. The following result is true for $L O$ as well as $E F$-proximities.

(1.4) Lemma. Let $f:\left(X, \delta_{\lambda}\right) \rightarrow\left(Y, \delta_{\lambda}^{\prime}\right)$ be p-continuous for each member $\lambda$ of some indexing set 1 . Then $f:\left(X, \bigvee \delta_{\lambda}\right) \rightarrow\left(Y, \bigvee \delta_{\lambda}^{\prime}\right)$ is p-continuous.

Proof. Let $\beta_{1}=\bigcap\left\{\delta_{\lambda}: \lambda \in I\right\}$ and $\beta_{2}=\bigcap\left\{\delta_{\lambda}^{\prime}: \lambda \in I\right\}$. Then for each $(A, B) \in \beta_{1},(f(A), f(B)) \in \beta_{2}$ and the conclusion follows from Lemma 1.3.

(1.5) Lemma. Let $F$ be a family of functions, each member $f$ of $F$ being on a set $X$ into a LO-space $\left(Y_{f}, \delta_{f}\right)$. Then there exists a coarsest LOproximity $\delta_{F}$ on $X$ making each member of $F$ p-continuous. Moreover $\delta_{F}$ is compatible with the weak topology on $X$ determined by the family $F$; and if each $\delta_{f}$ is $E F$ then so is $\delta_{F}$.

Proof. Define a binary relation $\beta_{F}$ on the power-set of $X$ as follows: $(A, B) \in \beta_{F}$ if and only if $(f A, f B) \in \delta_{f}$ for each $f$ in $F . \beta_{F}$ is a $L O$ base on $X$ and the $L O$-proximity $\delta_{F}=\delta\left(\beta_{F}\right)$ fulfills all the requirements.

The relation $\delta_{F}$ is called the weak-LO (respectively weak-EF) proximity on $X$ determined by the family $F$. By $\mathbf{L O}$ (respectively EF) we denote the category of $L O$-spaces (respectively $E F$-spaces) and $p$-continuous maps. $\mathrm{S}$ is the category of sets. 


\section{Topological functors.}

(2.1) Theorem. The functors (LO - S) and $(\mathbf{E F}-\mathbf{S})$ are absolutely topological.

Proof. Let $\left(Y_{i}, \delta_{i}\right)_{I}$ be a family of $L O$-spaces and $\left(X, f_{i}: X \rightarrow Y_{i}\right)_{I}$ be any source in $\mathrm{S}$. Denote by $\delta_{F}$ the weak $L O$-proximity on $X$ determined by the family $F=\left(f_{i}\right)_{I}$. Let $(Z, \delta)$ be a $L O$-space and suppose $g:(Z, \delta) \rightarrow$ $\left(X, \delta_{F}\right)$ is not $p$-continuous. Take $(A, B) \in \delta$ such that $(g A, g B) \notin \delta_{F}$. Since $\delta_{F}=\delta\left(\beta_{F}\right)$ there exist finite covers $\left\{H_{i}: 1 \leqq i \leqq m\right\}$ and $\left\{K_{j}: 1 \leqq j \leqq n\right\}$ of $g(A)$ and $g(B)$ respectively such that $\left(H_{i}, K_{j}\right) \notin \beta_{F}$ for any $i, j$. Also there exist $i, j$ such that $\left(g^{-1} H_{i}, g^{-1} K_{j}\right) \in \delta$; and as $\left(H_{i}, K_{j}\right) \notin \beta_{F}$ there is $f_{k} \in F$ such that $\left(f_{k} H_{i}, f_{k} K_{j}\right) \notin \delta_{k}$. This shows that $f_{k} g$ is not $p$-continuous. The proof is now complete.

(2.2) Theorem. For any full epireflective subcategory $\mathrm{C}$ of $\mathbf{L O}$ the functor $(\mathbf{C}-\mathrm{S})$ is (epi, mono-source) topological.

Proof. Let $\left(Y_{i}\right)_{I}$ be a family of objects in $\mathrm{C}$ and let $\left(X, m_{i}: X \rightarrow Y_{i}\right)_{I}$ be a mono-source in $\mathrm{S}$. Denote by $\delta_{I}$ the weak $L O$-proximity on $X$ induced by the family $\left\{m_{i}: i \in I\right\}$. The evaluation map on $\left(X, \delta_{I}\right)$ is a proximal isomorphism into the product of $\left(Y_{i}\right)_{I}$. Thus, $\left(X, \delta_{I}\right)$ belongs to $\mathrm{C}$. Obviously, the source $\left(\left(X, \delta_{I}\right), m_{i}\right)$ is initial to $\left(X, m_{i}\right)$ in $\mathbf{S}$ and the proof is complete.

By $\mathbf{L O}^{*}$ (respectively $\mathbf{E F}^{*}$ ) we denote the full subcategory of $\mathbf{L O}$ consisting of all separated $L O$ (respectively separated $E F$ ) spaces. Since LO*, $\mathbf{E F}$, and $\mathbf{E F}^{*}$ are full epireflective subcategories of $\mathbf{L O}$, we have the following:

(2.3) Corollary. Each of the functors $\left(\mathbf{L O}^{*}-\mathrm{S}\right),(\mathbf{E F}-\mathrm{S})$ and $\left(\mathbf{E F}^{*}-\mathrm{S}\right)$ is (epi, mono-source) topological.

(2.4) Example. The functor $\mathrm{LO}^{*}-\mathrm{S}$ is not absolutely topological.

Proof. Let $X$ be an infinite set. Take $X \times X=Z$ with the $L O$-proximity $\delta_{1}$ defined by $(P, Q) \in \delta_{1}$ if and only if $P \cap Q \neq \varnothing$ or each of the sets $P$ and $Q$ is infinite. Consider the function $\pi: Z \rightarrow X$ defined by $\pi\left(x_{1}, x_{2}\right)=x_{1}$ for all $\left(x_{1}, x_{2}\right) \in Z$. Then for any two disjoint infinite subsets $A$ and $B$ of $X$ we have $(b \times A, a \times B) \in \delta_{1}$, for $b \in B$ and $a \in A$; but $(b, a) \notin \delta$ for any separated $L O$-proximity $\delta$ on $X$. Thus no object in $\mathbf{L O}^{*}$ can be initial to $\left(X, f_{0}: X \rightarrow X\right)$ where $f_{0}$ is a constant function on $X$ to $X$.

We remark that none of the functors $\left(\mathbf{L O}-\mathbf{R}_{0}\right),\left(\mathbf{L O} \mathbf{O}^{*}-\mathrm{T}_{1}\right),(\mathbf{E F}-\mathbf{C R})$ $\left(\mathbf{E F}^{*}-\mathbf{T}_{31 / 2}\right)$ is absolutely topological. This can be easily seen by considering such examples as the following. 
(2.5) Example. For any nonindiscrete $R_{0}$-space $X$ there exists a $L O$ space $Z$ and a continuous function $f$ on $Z$ to $X$ such that for no compatible $L O$-proximity on $X$ is $f p$-continuous.

Proof: Let $Y$ be an infinite discrete topological space and set $Z=$ $X \times Y$. The projection $p_{x}$ of $Z$ into $X$ is continuous. Let $\delta_{c}$ be the coarsest compatible $L O$-proximity on $Z$. Since $X$ is nonindiscrete $R_{0}$, there exist two points $x_{1}, x_{2}$ of $X$ such that $\operatorname{cl}\left(\left\{x_{1}\right\}\right) \cap \operatorname{cl}\left(\left\{x_{2}\right\}\right)=\varnothing$, and therefore for any compatible $L O$-proximity $\delta$ on $X,\left(\left\{x_{1}\right\},\left\{x_{2}\right\}\right) \notin \delta$. Also $p_{x}^{-1} \operatorname{cl}\left(\left\{x_{1}\right\}\right)=$ $\left\{(a, y): a \in \operatorname{cl}\left(\left\{x_{1}\right\}\right), y \in Y\right\}$ and thus each of the sets $p_{x}^{-1} \operatorname{cl}\left(\left\{x_{1}\right\}\right)$, $p_{x}^{-1} \operatorname{cl}\left(\left\{x_{2}\right\}\right)$ is the union of infinitely many pairwise disjoint point-closures, thereby showing that $\left(p_{x}^{-1} \operatorname{cl}\left(\left\{x_{1}\right\}\right), p_{x}^{-1} \operatorname{cl}\left(\left(x_{2}\right\}\right)\right) \in \delta_{c}$. Thus the function $p_{x}$ cannot be $p$-continuous.

Let $X$ be a topological space. A determinator on $X$ is a source $\left(X, f_{i}\right)$ in TOP such that for each closed set $A$ in $X$ and each point $x$ in the complement of $A$ we can find a finite subfamily $\left\{f_{i}: 1 \leqq i \leqq n\right\}$ of $F$ and a finite cover $\left\{A_{i}: 1 \leqq i \leqq n\right\}$ of $A$ such that $f_{i}(x) \notin \operatorname{cl}\left(f_{i}\left(A_{i}\right)\right)$ for any $i$. Observe that any family of continuous functions which distinguishes points and closed sets is a determinator on $X$.

(2.6) Theorem. The weak topology determined on a topological space $X$ by a source of continuous functions on $X$ coincides with the given topology of $X$ if and only if the source is a determinator on $X$.

(2.7) Theorem. Let $\left(Y_{i}, \delta_{i}\right)$ be a collection of separated LO-spaces and $\left(X, f_{i}: X \rightarrow Y_{i}\right)$ a mono-source in $\mathrm{T}_{1}$. A source initial to $\left(X, f_{i}\right)_{1}$ via the functor $\left(\mathbf{L O}^{*}-\mathbf{T}_{1}\right)$ exists in $\mathbf{L} \mathbf{0}^{*}$ if and only if $F=\left(X, f_{i}\right)_{I}$ is a determinator on $X$.

Proof. If $F$ is a determinator on $X$ then by Lemma 1.5 and Theorem 2.6, the weak $L O$-proximity $\delta_{F}$ on $X$ is compatible (and separated), and thus the LO-space $\left(X, \delta_{F}\right)$ is initial to $\left(X, f_{i}\right)_{I^{\bullet}}$.

To prove the converse, suppose $F$ is not a determinator on $X$. Since each member of $F$ is continuous, $\delta_{F}$ must be coarser than the finest compatible $L O$-proximity on $X$. In fact the topology $\tau\left(\delta_{F}\right)$ induced by $\delta_{F}$ must be strictly coarser than the given topology, say $\tau$, on $X$. So there is a subset $A_{0}$ of $X$ such that $A_{0}$ is closed in $(X, \tau)$ but not closed in $\left(X, \tau\left(\delta_{F}\right)\right)$. Let $\delta_{c}$ be the coarsest $L O$-proximity on $X$ compatible with $\tau$ and set $\delta^{*}=$ $\delta_{c} \vee \delta_{F}$. It is clear that no object except perhaps $\left(X, \delta^{*}\right)$ could be initial to $\left(X, f_{i}, Y_{i}\right)$. But $\left(X, \delta^{*}\right)$ also fails to be initial to $\left(X, f_{i}, Y_{i}\right)$ for the 
following reason: We can find a separated $L O$-space $(Z, \delta)$ and a continuous function $g:(Z, \tau(\delta)) \rightarrow(X, \tau)$ such that $g:(Z, \delta) \rightarrow\left(X, \delta_{F}\right)$ is p-continuous but $g:(Z, \delta) \rightarrow\left(X, \delta_{c}\right)$ is not $p$-continuous and hence $g:(Z, \delta) \rightarrow\left(X, \delta^{*}\right)$ is not p-continuous. The space $(Z, \delta)$ is constructed as follows:

Let $A_{0}$ be given the subspace topology inherited from the topology $\tau$ on $X$, and let $Z$ be the disjoint topological union of $A_{0}$ and $N$ where $N$ is the discrete space of natural numbers. Since $A_{0}$ is not closed in the topology $\tau\left(\delta_{F}\right)$ on $X$ there is a point $x_{0} \notin A_{0}$ such that $x_{0}$ is in the closure of $A_{0}$ with regard to the topology $\tau\left(\delta_{F}\right)$ on $X$.

Define $g: Z \rightarrow X$ by $g(z)=z$ if $z \in A_{0}$ and $g(z)=x_{0}$ otherwise. Then $g: Z \rightarrow(X, \tau)$ is continuous. Let $\delta_{g}$ be the $L O$-proximity on $Z$ defined by $(A, B) \in \delta_{g}$ if and only if $(g A, g B) \in \delta_{F}$ and let $\delta_{1}$ be the coarsest compatible $L O$-proximity on $Z$. Set $\beta=\delta_{g} \cap \delta_{1}, \beta$ is a $L O$-base on $Z$ and $g:(Z, \delta(\beta)) \rightarrow\left(X, \delta_{F}\right)$ is p-continuous. Also if $r_{0}$ is the topology on $Z$ induced by $\delta(\beta)$ then $g:\left(Z, \tau_{0}\right) \rightarrow(X, \tau)$ is continuous. Now we complete the proof by showing that $g:(Z, \delta(\beta)) \rightarrow\left(X, \delta_{c}\right)$ is not $p$-continuous. Since each of the sets $A_{0}$ and $N$ are infinite, it is clear that $\left(A_{0}, N\right) \in \beta$. Let $\left\{P_{i}: 1 \leqq i \leqq m\right\}$ and $\left\{N_{j}: 1 \leqq j \leqq n\right\}$ be any finite covers of $A_{0}$ and $N$ respectively. Then there exists $i_{0}$, such that $P_{i_{0}}$ is infinite and $x_{0}$ is in the $\tau\left(\delta_{F}\right)$-closure of $g\left(P_{i_{0}}\right)=P_{i_{0}}$. Also there is a $j_{0}$ such that $N_{j_{0}}$ is infinite. Therefore $\left(P_{i_{0}}, N_{j_{0}}\right) \in \beta$ and hence $\left(A_{0}, N\right) \in \delta(\beta)$. This shows that $g:(Z, \delta(\beta)) \rightarrow\left(X, \delta_{c}\right)$ is not $p$-continuous and completes the proof.

An $E F$-space $(X, \delta)$ is called p-stable if and only if the p-class $\Pi(\delta)$ of uniformities on $X$ compatible with $\delta$ has a finest member. In what follows, $\mathbf{U}$ denotes the category of uniform spaces and uniformly continuous maps.

(2.8) Theorem. An EF-space $(X, \delta)$ is p-stable if and only if for each source $\left((X, \delta), f_{i}\right)_{I}$ in $E F$, there exists a source initial to it in $\mathbf{U}$.

Proof. Suppose that $\delta$ is $p$-stable and let $\mathcal{U}_{1}$ and $\mathcal{U}_{0}$ be respectively the finest and the coarsest member of the p-class $\Pi(\delta)$ of uniformities compatible with $\delta$. Let $\mathcal{U}_{F}$ be the weak uniformity determined on $X$ by $F=$ $\left(f_{i}\right)_{I}$. Since each $f \in F$ is p-continuous, each member of $F$ is uniformly continuous if the uniformity $\mathcal{U}_{1}$ is taken on $X$. Therefore $\mathcal{U}_{F} \subseteq \mathcal{U}_{1}$. Let $\mathcal{U}^{*}=$ $\mathcal{U}_{F} \vee \mathcal{U}_{0}$. Then $\mathcal{U}^{*}$ is compatible with $\delta$ and each member of $F$ is uniformly continuous if we take the uniformity $\mathcal{U}^{*}$ on $X$. In fact $\mathcal{U}^{*}$ is the coarsest uniformity on $X$ compatible with $\delta$ such that each function in $F$ becomes uniformly continuous. Now we claim that $\left(X, \mathrm{U}^{*}\right)$ is initial to the given source. Suppose $(Z, \mathcal{U})$ is a uniform space such that $(i) g:(Z, \mathcal{U}) \rightarrow(X, \delta)$ 
is $p$-continuous, and (ii) $f \cdot g$ is uniformly continuous for each $f$ in $F$. The proximal continuity of $g$ implies that $g:(Z, \mathcal{U}) \rightarrow\left(X, \mathcal{U}_{\mathbb{U}}\right)$ is uniformly continuous, and the fact that $f \cdot g$ is uniformly continuous for each $f$ in $F$ implies that $g:(Z, \mathcal{U}) \rightarrow\left(X, \mathcal{U}_{F}\right)$ is uniformly continuous. From these we conclude that $g:(Z, \mathcal{U}) \rightarrow\left(X, \mathcal{U}^{*}\right)$ is uniformly continuous.

Now to prove the converse, we suppose that $(X, \delta)$ is not $p$-stable. Let $\left\{\mathcal{U}_{\lambda}: \lambda \in \Lambda\right\}$ be the $p$-class of uniformities of $\delta$. For each $\lambda \in \Lambda$ take $I_{\lambda}: X \rightarrow$ $\left(X, \mathcal{U}_{\lambda}\right)$ defined by $I_{\lambda}(x)=x$ for all $x \in X$ and set $F=\left\{I_{\lambda}: \lambda \in \Lambda\right\}$. Since there is no finest member in the collection $\left\{\mathcal{U}_{\lambda}: \lambda \in \Lambda\right\}$ it follows that $(X, \delta)$ cannot be lifted to an initial source.

It would be interesting to have a characterization of those sources which the functor $\left(\mathbf{U}-\mathbf{T}_{3 \frac{1}{2}}\right)$ lifts to initial sources.

\section{BIBLIOGRAPHY}

1. M. S. Gagrat and S. A. Naimpally, Proximity approach to extension problems, Fund. Math. 71 (1971), 63-76. MR 45 \#2653.

2. H. Herrlich, Topological functors, General Topology and Appl. (to appear).

3. H. Herrlich and G. Strecker, Category theory, Allyn and Bacon, Boston, Mass., 1973.

4. P. L. Sharma and S. A. Naimpally, Construction of Lodato proximities, Math. Japon. 15 (1970), 101-103. MR 44 \#4709.

5. P. L. Sharma, Proximity bases and subbases, Pacific J. Math. 37 (1971), 515-526. MR 46 \#4488.

DEPARTMENT OF MATHEMATICS, SOUTHERN ILLINOIS UNIVERSITY, CARBONDALE, ILLINOIS 62901 